\title{
Motion planning in quantum control via intersection of eigenvalues
}

\author{
Ugo Boscain, Francesca C. Chittaro, Paolo Mason, Rémi Pacqueau and Mario Sigalotti
}

\begin{abstract}
In this paper we consider the problem of inducing a transition in a controlled quantum mechanical system whose spectrum loses simplicity for some values of the control. We study the situation in which the Hamiltonian of the system is real, and we are in presence of two controls. In this case, eigenvalue crossings are generically conical. Adiabatic approximation is used to decouple a finite dimensional subsystem from the original one (usually infinite dimensional).

The main advantage of this method is that as a byproduct of the controllability result it permits to get an explicit expression of the controls. Moreover it may be used in the case in which the dependence of the Hamiltonian from the controls is non-linear, for which at the moment, no other method works.

In this paper we study the basic block of this controllability method, that is a two by two system whose spectrum presents a conical intersection. We show how to control exactly this system with a control strategy that can be slowed down. The possibility of slowing down the control law is essential to obtain an adiabatic decoupling from the rest of the system with an arbitrary precision.
\end{abstract}

Keywords: Quantum control, Adiabatic approximation.

\section{INTRODUCTION}

In the simplest case, the problem of controlling a quantum mechanical system consists in controlling an equation of the form

$$
i \dot{\psi}=\left(H_{0}+\sum_{i=1}^{m} u_{i} H_{i}\right) \psi
$$

where $\psi$ belongs to the unit sphere in a Hilbert space $\mathcal{H}$, the self-adjoint operator $H_{0}$ describes the free evolution of the system, $H_{i}, i=1, \ldots, m$ are self-adjoint operators describing the coupling between the controls and the system, and $u_{i}:[0, T] \rightarrow \mathbb{R}$ are the controls, usually representing an electric field, a magnetic field or a laser pulse.

Nowadays quantum control is of crucial importance in Nuclear Magnetic Resonance (NMR), in photochemistry and

U. Boscain is with CNRS CMAP, École Polytechnique, Palaiseau, France ugo.boscainepolytechnique.edu

\footnotetext{
F. C. Chittaro is with L2S-Supélec francesca.chittaro@lss.supelec.fr

P. Mason is with CNRS-L2S-Supélec paolo.masonalss.supelec.fr

R. Pacqueau is with CNRS CMAP, École Polytechnique, Palaiseau, France remi.pacqueau@polytechnique.edu

M. Sigalotti is with INRIA Nancy - Grand Est, Équipe-projet CORIDA, mario.sigalotti@inria.fr

${ }^{1}$ This research has been supported by the European Research Council, ERC StG 2009 "GeCoMethods", contract number 239748, by the ANR "GCM", program "Blanc - CSD", and by the DIGITEO project "CONGEO".
}

for the design of quantum gates in quantum computing (see [17], [23] [21], and references therein).

Many results are available when the Hilbert space has finite dimension (see for instance [2], [3], [4], [9], [8], [7], [12], [14], [15], [18], [22], and the monograph [13]). On the other hand in the infinite dimensional case positive controllability results are extremely difficult to obtain. For exact controllability results for a one-dimensional well of potential see [5]. For approximate controllability results for discrete spectrum, via Galerkin approximations, see [10]. For approximate controllability results via Lyapunov methods, see [20], [19]. Notice that except for the results given in [10], most of the controllability results are obtained for systems in the form

$$
i \partial_{t} \psi(t, x)=\left(-\Delta+V_{0}(x)+\sum_{i=1}^{m} u_{i} V_{i}(x)\right) \psi(t, x)
$$

where $x \in \Omega \subset \mathbb{R}^{n}, \Delta$ is the Laplacian (with Dirichlet boundary conditions, when $\Omega$ is bounded), $V_{0}$ is the potential in absence of controls, and $V_{i}$ are suitable control potentials.

For infinite dimensional systems, the need of generating an infinite number of new directions requires highly oscillating controls, and usually the techniques mentioned above do not lead to explicit control strategies.

Another approach could be to design a slow control path that passes through eigenvalues intersections in the space of controls allowing exchanges of probability between the intersecting levels (in this case, we say that the wave function climbs the spectrum). This idea was presented for the first time in [1] for a couple of specific systems. To prove that such a path indeed produces a transition, one should: i) take advantage of the adiabatic decoupling far from eigenvalues crossing in the space of controls; ii) use another nonadiabatic decoupling (that we call conical decoupling) close to eigenvalues intersections that permits to prove that, while passing through eigenvalue intersections, the system makes a transition from a level to another.

The first step is quite standard in quantum mechanics (see for instance [24]), while the second one will be explained in full details below. The main feature that makes this method work is the fact that, as a consequence of the self-adjointness of the operators $H_{0}$ and $H_{i}, i=1 \ldots m$, the codimension of the eigenvalues intersections is bigger than one. For instance in the case in which the Hamiltonian is real, the codimension of eigenvalues intersections is two and hence, in the presence of two controls, generically they occur at isolated points in the space of controls. Since climbing of levels can be realized only at eigenvalue crossings, thanks to the fact that 
the intersections have codimension two we can construct closed paths that pass through each crossing only once, and therefore realize the climbing; see the red path in Figure 1. Transversal eigenvalue crossings of a real Hamiltonian in the presence of two controls are called conical intersections (see [11]). A non-real Hamiltonian can have eigenvalue intersections which are stable under small perturbations of the Hamiltonian and are transversal at isolated points only if it depends on at least three controls.

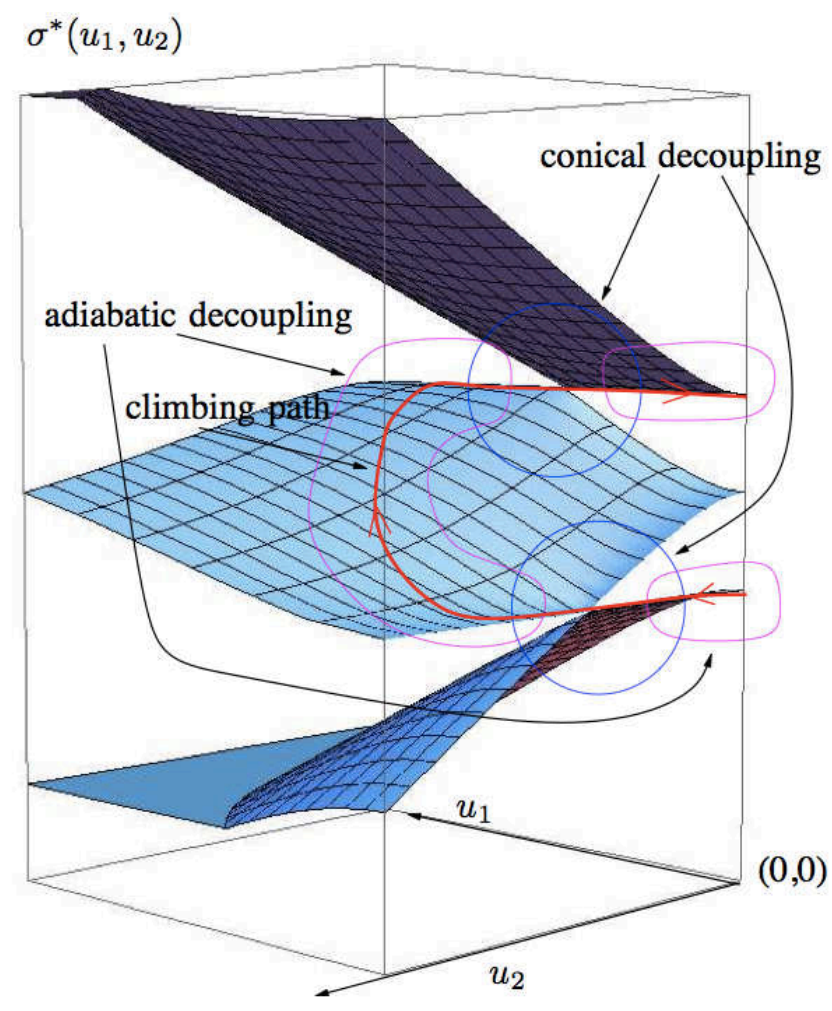

Fig. 1. Climbing path $u$.

This approach has already been explored in [16], [25] for the STIRAP process. From the controllability point of view, this approach has been applied in [1] to a class of models generalizing the Eberly and Law system [6].

This method has two advantages. First it provides explicit expressions of controls (motion planning); second it may be applied without difficulties also in the case in which the dependence on controls is nonlinear. The drawback is that the method works only when one can produce eigenvalue crossings via the external fields.

Let $u=\left(u_{1}, \ldots, u_{m}\right)$ and $\sigma(u)$ be the spectrum of $H(u)$. In the following we do not need that the dependence on $u$ is affine as in (1). Let $\sigma^{*}(u) \subset \sigma(u)$ be a band that is uniformly separated from the rest of the spectrum in a connected domain $U$ in the space of controls (see Figure 2) and $P^{*}(u)$ the corresponding orthogonal projector. Roughly speaking, the Adiabatic Theorem (see [24] and references therein) states that if $u:[0, T] \rightarrow \mathbb{R}^{m}$ is a smooth control and $\psi_{u}^{\varepsilon}$ is a trajectory of system (1), corresponding to the control $[0, T / \varepsilon] \ni t \mapsto u(\varepsilon t)$ and such that $\psi_{u}^{\varepsilon}(0)=\psi_{0} \in P^{*}(u) \mathcal{H}$, then as $\varepsilon$ gets small, $\psi_{u}^{\varepsilon}$ on $[0, T / \varepsilon]$ belongs approximately to $P^{*}(u) \mathcal{H}$ and its evolution is not influenced by the rest of the spectrum. The quality of the approximation depends on the "gap" $C$ between $\sigma^{*}(u)$ and the rest of the spectrum. Roughly speaking the error is proportional to $1 / C$.

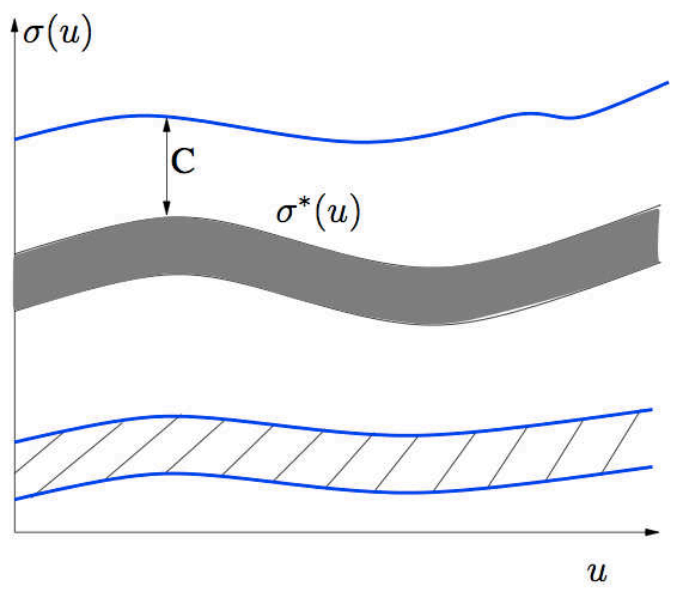

Fig. 2. Spectrum in function of $u$.

When $P^{*}(u) \mathcal{H}$ is finite dimensional, the adiabatic theory permits also to build a finite dimensional Hamiltonian $H_{\text {eff }}$, describing the approximate evolution inside $P^{*}(u) \mathcal{H}$. The precise construction of $H_{\text {eff }}$ as function of $u$ will be presented in a forthcoming paper, and is based on general adiabatic theory ([24]).

In this paper we are interested in controllability problems inside the finite dimensional space $P^{*}(u) \mathcal{H}$, for a system governed by an Hamiltonian $H_{\text {eff }}(u)$. We are then looking for control strategies that can be slowed down (in order to guarantee the adiabatic decoupling with arbitrary precision from the rest of the spectrum). Notice that we are not interested in controlling relative phases, since the adiabatic approximation loses any information about them.

\section{Slow CONTROLlability IN FINITE DimENSION}

We are ready to state our definition of controllability inside the space $P^{*}(u) \mathcal{H} \simeq \mathbb{C}^{N}$.

Definition 1: Consider a Schrödinger equation in $\mathbb{C}^{N}$ of the type

$$
i \dot{\psi}=H(u) \psi
$$

where $\psi(t) \in \mathbb{C}^{N}, u:[0, T] \rightarrow U \subset \mathbb{R}^{m}$, and $H(u)$ is a self-adjoint operator on $\mathbb{C}^{N}$, which is smooth as a 
function of $u$. We say that the system is slowly controllable between probabilities (SCBP for short) in $\bar{U} \subset U$ if for every $c, d \in[0,1]^{N}$ such that $\sum_{j} c_{j}=1, \sum_{j} d_{j}=1$, and for every $\bar{u} \in \bar{U}$, there exists a control function $u(t)=\left(u_{1}(t), \ldots, u_{m}(t)\right):[0, T] \rightarrow \bar{U}$, continuous and piecewise smooth with $u(0)=u(T)=\bar{u}$, such that the following holds: for every $\psi^{0}$ such that $\left(\left|\psi_{1}^{0}\right|^{2}, \ldots,\left|\psi_{N}^{0}\right|^{2}\right)=$ $c$ there exists a sequence of continuous, piecewise smooth monotone functions $\mu_{i}:\left[0, T_{i}\right] \rightarrow[0, T], T=\mu_{i}\left(T_{i}\right)$, with $\lim _{i \rightarrow \infty}\left\|\dot{\mu}_{i}(t)\right\|_{\infty}=0$ such that for every $i \in \mathbf{N}$ the control $\left[0, T_{i}\right] \ni t \mapsto u\left(\mu_{i}(t)\right)$ steers $\psi^{0}$ to some vector $\psi\left(T_{i}\right)$ with $\left(\left|\psi_{1}^{1}\right|^{2}, \ldots,\left|\psi_{N}^{1}\right|^{2}\right)=d$.

Remark 1: In the previous definition, $\mu_{i}(t)$ are reparametrizations of the time. For $i \rightarrow \infty$ the intervals $\left[0, T_{i}\right]$ become larger and larger. In our construction the rescalings can be taken piecewise-affine (although in general not linear).

If the selected spectrum $\sigma^{*}(u) \subset \sigma(u)$ is discrete and non-degenerate, then SCBP never holds unless we are in the trivial case $N=1$. Indeed an application of the adiabatic theorem inside $P^{*}(u) \mathcal{H}$ does not permit any probability transfer.

We then consider the case in which there are eigenvalue intersections in the space $U$ : as we will show, every intersection allows transfers of probability between the corresponding two energy levels. That is why we focus on a neighborhood of a conical eigenvalue crossing: the basic block of the algorithm consists in studying the controllability of a twolevel system driven by two controls (see Figure 1).

We prove that, close to a conical intersection, a two level system is SCBP with explicit computations, without any adiabatic approximation.

The general model for such a system with two controls is

$$
i \dot{\psi}=H\left(u_{1}(t), u_{2}(t)\right) \psi
$$

where $\psi=\left(\psi_{1}, \psi_{2}\right):[0, T] \rightarrow \mathbb{C}^{2}$ with $\left|\psi_{1}\right|^{2}+\left|\psi_{2}\right|^{2}=1$, $u_{1}, u_{2}:[0, T] \rightarrow \mathbb{R}$ and $H\left(u_{1}, u_{2}\right)$ is a real-valued matrix of the form

$$
H\left(u_{1}, u_{2}\right)=\left(\begin{array}{cc}
f_{1}\left(u_{1}, u_{2}\right) & f_{2}\left(u_{1}, u_{2}\right) \\
f_{2}\left(u_{1}, u_{2}\right) & -f_{1}\left(u_{1}, u_{2}\right)
\end{array}\right) .
$$

In the following we assume that $f_{1}$ and $f_{2}$ are smooth.

Remark 2: In formula (4) we have assumed that the trace is zero. This is not restrictive, since a nonvanishing trace gives rise only to a common factor of phase.

The eigenvalues of the matrix $H\left(u_{1}, u_{2}\right)$ are

$$
\lambda_{ \pm}= \pm \sqrt{f_{1}\left(u_{1}, u_{2}\right)^{2}+f_{2}\left(u_{1}, u_{2}\right)^{2}} .
$$

Hence a degeneration occurs at $\left(\bar{u}_{1}, \bar{u}_{2}\right)$ if and only if

$$
f_{1}\left(\bar{u}_{1}, \bar{u}_{2}\right)=f_{2}\left(\bar{u}_{1}, \bar{u}_{2}\right)=0 .
$$

Notice that for a generic choice of $f_{1}$ and $f_{2}$, the points at which the eigenvalues lose simplicity are isolated in the plane $\left(u_{1}, u_{2}\right)$.

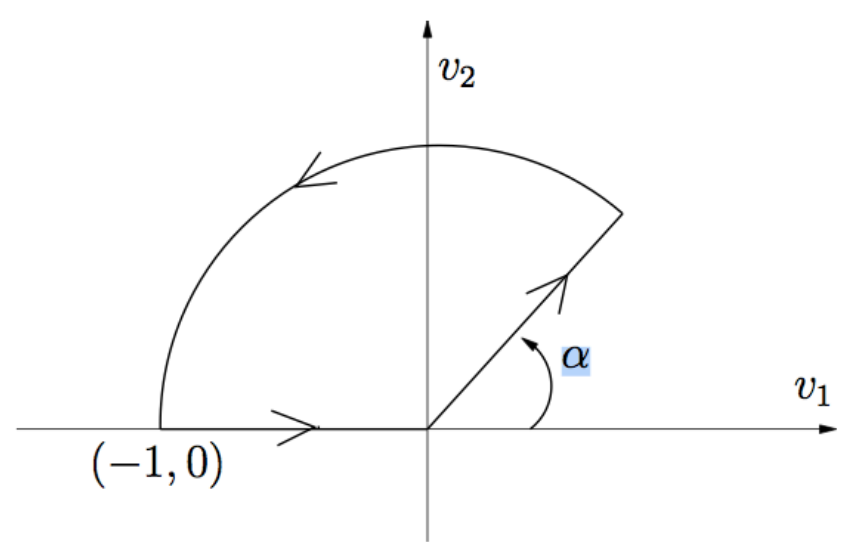

Fig. 3. The path in the plane $\left(v_{1}, v_{2}\right)$.

Theorem 1: Consider a two-level quantum system

$$
i \dot{\psi}=H\left(u_{1}(t), u_{2}(t)\right) \psi
$$

where $\psi=\left(\psi_{1}, \psi_{2}\right):[0, T] \rightarrow \mathbb{C}^{2}$ with $\left|\psi_{1}\right|^{2}+\left|\psi_{2}\right|^{2}=1$, $u_{1}, u_{2}:[0, T] \rightarrow U \subset \mathbb{R}$ and $H\left(u_{1}, u_{2}\right)$ is a real-valued matrix of the form (4). Assume that $\operatorname{det}\left(H\left(\bar{u}_{1}, \bar{u}_{2}\right)\right)=$ 0 and that the differential of the function $\left(u_{1}, u_{2}\right) \mapsto$ $\left(f_{1}\left(u_{1}, u_{2}\right), f_{2}\left(u_{1}, u_{2}\right)\right)$ is invertible at $\left(\bar{u}_{1}, \bar{u}_{2}\right)$. Then the system is SCBP in a neighborhood of $\left(\bar{u}_{1}, \bar{u}_{2}\right)$.

Here we sketch the explicit strategy we intend to use. We perform the change of coordinates

$$
\left(v_{1}, v_{2}\right)=F\left(u_{1}, u_{2}\right):=\rho\left(f_{1}\left(u_{1}, u_{2}\right), f_{2}\left(u_{1}, u_{2}\right)\right),
$$

$\rho>0$, from a neighborhood of $\left(\bar{u}_{1}, \bar{u}_{2}\right)$ to an open set containing the unit ball; we then construct a closed oriented path in the space of controls $\left(v_{1}, v_{2}\right)$ that is piecewise smooth and passes through the conical intersection (the origin in these coordinates); we choose a path that possesses the graph shown in Figure 3: we go straight from the starting point $(-1,0)$ to the singularity, then we make an angle $\alpha$ and continue with another segment, and finally we come back to the starting point with an arc of circle. Assuming without loss of generality that $\rho=1$ (this corresponds to a simple time reparametrization), this path can be realized as the graph of the following function:

$$
\left(v_{1}, v_{2}\right): t \mapsto \begin{cases}\left(\frac{t}{L}-1,0\right) & t \in[0, L] \\ (\cos \alpha, \sin \alpha)\left(\frac{t}{L}-1\right) & t \in[L, 2 L] \\ (\cos (\theta(t)), \sin (\theta(t))) & t \in[2 L, T]\end{cases}
$$

where

$$
L=\frac{T}{2+\pi-\alpha}, \quad \theta(t)=\alpha+\frac{t-2 L}{L} .
$$

This function is indeed continuous and piecewise smooth, and satisfies the condition $\left(v_{1}(0), v_{2}(0)\right)=\left(v_{1}(T), v_{2}(T)\right)$.

We will prove that the controls $\left(u_{1}(t), u_{2}(t)\right)=$ $F^{-1}\left(v_{1}(t), v_{2}(t)\right)$ guarantee SCBP, in the following sense: 
for every $c, d$ probabilistic weights there is an $\alpha \in[0, \pi]$ (depending only on $c, d$ ) such that for any $\psi^{0}=\left(\psi_{1}^{0}, \psi_{2}^{0}\right)$ with $\left(\left|\psi_{1}^{0}\right|^{2},\left|\psi_{2}^{0}\right|^{2}\right)=\left(c_{1}, c_{2}\right)$ we can find a sequence of time rescalings $\mu_{i}^{\alpha}$ that satisfy the conditions in the definition of SCBP and such that the control $\left(v_{1}\left(\mu_{i}^{\alpha}(t)\right), v_{2}\left(\mu_{i}^{\alpha}(t)\right)\right)$ defined on the interval $\left[0, T_{i}\right]$ steers $\psi^{0}$ to a state $\psi^{1}=$ $\left(\psi_{1}^{1}, \psi_{2}^{1}\right)$ such that $\left(\left|\psi_{1}^{1}\right|^{2},\left|\psi_{2}^{1}\right|^{2}\right)=\left(d_{1}, d_{2}\right)$.

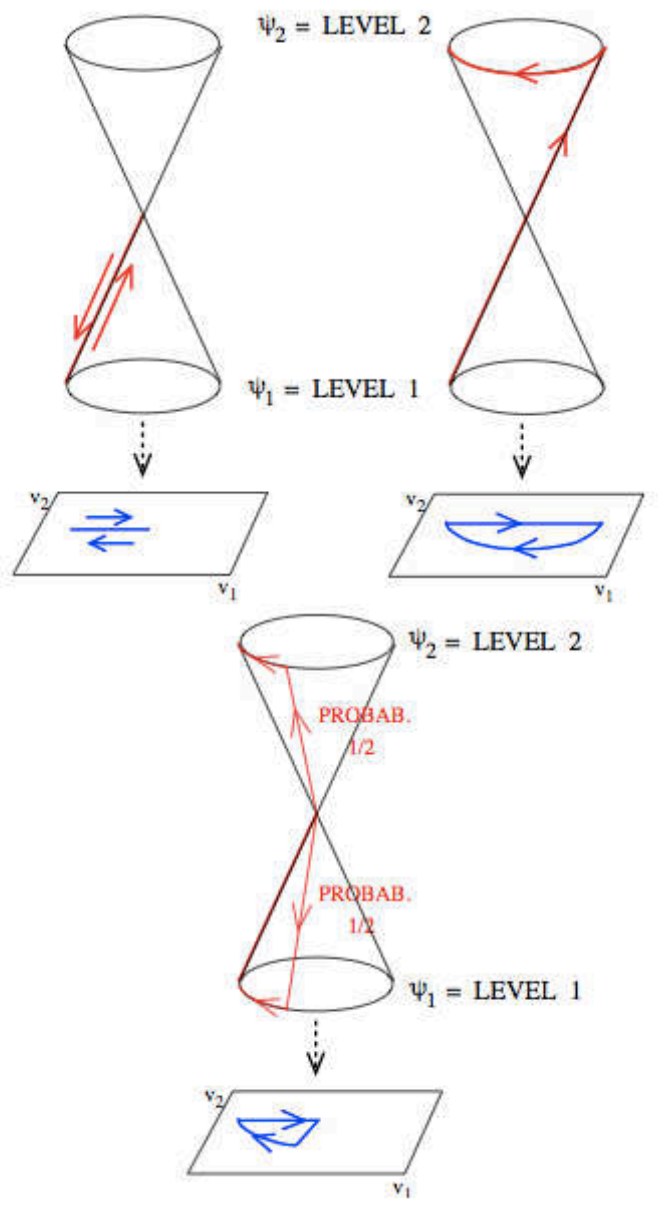

Fig. 4. Paths in the space of controls.

The angle $\alpha$ can be explicitly computed (see (10)); for instance, if $\psi^{0}=e^{i \phi}(1,0)$, then we have the following values (see Figure 4):

$$
\begin{gathered}
\left(\left|\psi_{1}^{1}\right|^{2},\left|\psi_{2}^{1}\right|^{2}\right)=(1,0) \Rightarrow \alpha=\pi \\
\left(\left|\psi_{1}^{1}\right|^{2},\left|\psi_{2}^{1}\right|^{2}\right)=(0,1) \Rightarrow \alpha=0 \\
\left(\left|\psi_{1}^{1}\right|^{2},\left|\psi_{2}^{1}\right|^{2}\right)=\left(\frac{1}{2}, \frac{1}{2}\right) \Rightarrow \alpha=\frac{\pi}{2} .
\end{gathered}
$$

The above control satisfies $u(0)=u(T)=\bar{u}:=$ $F^{-1}(-1,0)$. Control strategies for different $\bar{u}$ can be obtained from the one above, applying a rotation and a dilation in the plane $\left(v_{1}, v_{2}\right)$.

\section{SKeTCH OF THE PROOF}

\section{A. Normal form at an eigenvalue intersection}

Assume that $f_{1}\left(\bar{u}_{1}, \bar{u}_{2}\right)=f_{2}\left(\bar{u}_{1}, \bar{u}_{2}\right)=0$ and that the differential of the function $\left(u_{1}, u_{2}\right) \mapsto\left(f_{1}\left(u_{1}, u_{2}\right), f_{2}\left(u_{1}, u_{2}\right)\right)$ is invertible at $\left(\bar{u}_{1}, \bar{u}_{2}\right)$. Then, locally near $\left(\bar{u}_{1}, \bar{u}_{2}\right)$ we can perform the change of variables (7); up to a simple reparametrization of time, from now on we assume that $\rho=1$. We thus obtain the normal form

$$
i \dot{\psi}=\left(\begin{array}{cc}
v_{1} & v_{2} \\
v_{2} & -v_{1}
\end{array}\right) \psi
$$

whose eigenvalues are

$$
\lambda_{ \pm}= \pm \sqrt{v_{1}^{2}+v_{2}^{2}}
$$

\section{B. The control procedure}

In this subsection we solve explicitly the differential system (9) associated with the control (8) in the case in which the initial condition is an eigenvector of the Hamiltonian; we show how the choice of the angle $\alpha$ and of the ( $\alpha$-dependent) time reparametrization $\mu_{k}^{\alpha}$ let us control the probability weights of the wave function at the final time $\left(\mu_{k}^{\alpha}\right)^{-1}(T)$. The choice of such an initial condition gives rise to a family of reparametrizations $\mu_{k}^{\alpha}$ that is particularly simple (multiplication by a constant factor); for initial conditions that are nontrivial combinations of the two eigenvectors, to obtain SCBP one may still apply controls of the form (8), up to a suitable, possibly piecewise-linear, time reparametrization.

Let us now analyze the evolution of the system (9). We assume then that our initial state is $\psi^{0}=\left(e^{i \phi}, 0\right)$ (i.e. $c=$ $(1,0))$ and that we are given a pair $\left(d_{1}, d_{2}\right) \in[0,1]^{2}, d_{1}+$ $d_{2}=1$. We choose

$$
\alpha=2 \arcsin \left(\sqrt{d_{1}}\right) .
$$

First segment: Putting $\psi=\left(\psi_{1}, \psi_{2}\right)$, the system (9) evolves accordingly to the differential equation

$$
\dot{\psi}_{1}(t)=-i(t / L-1) \psi_{1}(t), \quad \dot{\psi}_{2}(t)=i(t / L-1) \psi_{2}(t),
$$

therefore the solution at the time $t$ is

$$
\psi(t)=\left(e^{-i\left(\frac{t^{2}}{2 L}-t\right)} \psi_{1}(0), e^{i\left(\frac{t^{2}}{2 L}-t\right)} \psi_{2}(0)\right) .
$$

If $\psi(0)=\psi^{0}$, we get $\psi(L)=\left(e^{i \phi_{1}}, 0\right), \phi_{1}=\phi+L / 2$. This means that the evolution only adds a (relative) phase factor, but does not change the probabilities.

Second segment: In this case we have to solve the Schrödinger equation

$i \frac{d}{d t}\left(\begin{array}{l}\psi_{1} \\ \psi_{2}\end{array}\right)=\left(\begin{array}{cc}\cos (\alpha)(t / L-1) & \sin (\alpha)(t / L-1) \\ \sin (\alpha)(t / L-1) & -\cos (\alpha)(t / L-1)\end{array}\right)\left(\begin{array}{l}\psi_{1} \\ \psi_{2}\end{array}\right)$

$t \in[L, 2 L]$, with initial condition $\psi(L)=\left(e^{i \phi_{1}}, 0\right)$. The solution of the Cauchy problem is

$$
\left(\begin{array}{l}
\psi_{1}(t) \\
\psi_{2}(t)
\end{array}\right)=e^{i \phi_{1}}\left(\begin{array}{c}
\cos \left(\phi_{2}(t)\right)-i \sin \left(\phi_{2}(t)\right) \cos (\alpha) \\
-i \sin \left(\phi_{2}(t)\right) \sin (\alpha)
\end{array}\right),
$$


where $\phi_{2}(t)$ is the dynamical phase $\phi_{2}(t)=\frac{t^{2}}{2 L}-t+\frac{L}{2}$.

In particular, we notice that for $\left(v_{1}, v_{2}\right)=$ $(\cos (\alpha), \sin (\alpha))$, the eigenvectors of the Hamiltonian are

$$
\begin{aligned}
& \varphi_{+}=(\cos (\alpha / 2), \sin (\alpha / 2)), \\
& \varphi_{-}=(-\sin (\alpha / 2), \cos (\alpha / 2)),
\end{aligned}
$$

respectively associated with the eigenvalues $\lambda=+1$ and -1 . Therefore, we can write the wave function at the time $t=2 L$ as

$\psi(2 L)=e^{i \phi_{1}}\left(e^{-i \phi_{2}(2 L)} \cos (\alpha / 2) \varphi_{+}+e^{i \phi_{2}(2 L)} \sin (\alpha / 2) \varphi_{-}\right)$.

We notice that the probability of being in an eigenstate relative to the eigenvalue $\lambda=+1$ is $(\cos (\alpha / 2))^{2}$; in other words, we spread the probability weights between the two eigenstates.

Arc of circumference: The last part of the path comes back to the initial point $\left(v_{1}, v_{2}\right)=(-1,0)$. The final state is then the solution at the time $T$ of the equation

$$
i \frac{d}{d t}\left(\begin{array}{l}
\psi_{1} \\
\psi_{2}
\end{array}\right)=\left(\begin{array}{cc}
\cos (\theta(t)) & \sin (\theta(t)) \\
\sin (\theta(t)) & -\cos (\theta(t))
\end{array}\right)\left(\begin{array}{l}
\psi_{1} \\
\psi_{2}
\end{array}\right)
$$

with initial condition $\psi(2 L)=e^{i \phi_{+}} \cos (\alpha / 2) \varphi_{+}+$ $e^{i \phi_{-}} \sin (\alpha / 2) \varphi_{-}$.

We claim that there is a suitable choice of time rescalings $\mu_{k}^{\alpha}$ such that the controls $\left(v_{1}\left(\mu_{k}^{\alpha}(t)\right), v_{2}\left(\mu_{k}^{\alpha}(t)\right)\right.$ defined on $\left[0,\left(\mu_{k}^{\alpha}\right)^{-1}(T)\right]$ steer the initial vector $\left(e^{i \phi}, 0\right)$ to the vector of the form

$$
\psi^{1}=e^{i \beta_{+}} \sin (\alpha / 2)\left(\begin{array}{l}
1 \\
0
\end{array}\right)+e^{i \beta_{-}} \cos (\alpha / 2)\left(\begin{array}{l}
0 \\
1
\end{array}\right),
$$

for suitable phases $\beta_{ \pm}$.

In order to do that, we study the system

$$
i \frac{d}{d t}\left(\begin{array}{l}
x_{1} \\
x_{2}
\end{array}\right)=\left(\begin{array}{cc}
\cos (\alpha+t / \tau) & \sin (\alpha+t / \tau) \\
\sin (\alpha+t / \tau) & -\cos (\alpha+t / \tau)
\end{array}\right)\left(\begin{array}{l}
x_{1} \\
x_{2}
\end{array}\right)
$$

for $t \in[0,(\pi-\alpha) \tau]$, where $\tau$ is a free parameter, with initial condition $x(0)=\left(x_{1}(0), x_{2}(0)\right)=\varphi_{+}$. We notice that this system is completely analogous to (11), up to an affine time transformation.

We evaluate $\left|x_{i}(t)\right|^{2}, i=1,2$, at the final time $t=(\pi-$ $\alpha) \tau$, as a function of $\tau$; explicit computations lead to

$$
\left|x_{2}(t)\right|^{2}=\frac{1}{4 \tau^{2}+1}\left[4 \tau^{2}+\cos \left(\frac{\pi-\alpha}{2} \sqrt{4 \tau^{2}+1}\right)^{2}\right] .
$$

Therefore there is a sequence of times $\tau_{k}^{\alpha}$, going to infinity, such that for $\tau=\tau_{k}^{\alpha}$ and $t_{k}^{\alpha}=(\pi-\alpha) \tau_{k}^{\alpha}$, we have $\left|x_{2}\left(t_{k}^{\alpha}\right)\right|^{2}=1$. Hence, from the unitarity of the evolution, $\left|x_{1}\left(t_{k}^{\alpha}\right)\right|^{2}=0$; analogously, for the initial condition $x(0)=$ $\varphi_{-}$we have $\left|x_{2}\left(t_{k}^{\alpha}\right)\right|^{2}=0$ and $\left|x_{1}\left(t_{k}^{\alpha}\right)\right|^{2}=1$.

For $\alpha \in[0, \pi)$ the explicit form of $\tau_{k}^{\alpha}$ is

$$
\tau_{k}^{\alpha}=\sqrt{\left(\frac{\pi}{\pi-\alpha}\right)^{2} k^{2}-\frac{1}{4}}
$$

while for $\alpha=\pi$ the equation is satisfied for any value of $\tau_{k}^{\alpha}$.

This means that after an evolution of time $t_{k}^{\alpha}$ the eigenvector of the Hamiltonian evaluated at $\left(v_{1}, v_{2}\right)=$ $(\cos (\alpha), \sin (\alpha))$ relative to the eigenvalue $\lambda=+1$ has moved to the vector $\psi=(0,1)$, which is in fact the eigenvector of the Hamiltonian at $\left(v_{1}, v_{2}\right)=(-1,0)$ relative to the same eigenvalue. Analogously, at the final time $\varphi_{-}$ has moved to $\psi=(1,0)$.

Coming back to the initial system, we have proved that if we choose the constant time rescaling

$$
\mu_{k}^{\alpha}: t \mapsto \frac{L}{\tau_{k}^{\alpha}} t, \quad t \in\left[0, \frac{\tau_{k}^{\alpha}}{L} T\right]
$$

then the chosen control $\left(v_{1}\left(\mu_{k}^{\alpha}(t)\right), v_{2}\left(\mu_{k}^{\alpha}(t)\right)\right)$, defined on $\left[0,\left(\mu_{k}^{\alpha}\right)^{-1}(T)\right]$, steers the initial state $\psi^{0}=\left(e^{i \phi}, 0\right)$ to the final state (12), which means that we steered the vector with probabilities $\left(\left|\psi_{1}^{0}\right|^{2},\left|\psi_{2}^{0}\right|^{2}\right)=(1,0)$ to a vector $\psi^{1}$ with probabilities $\left(\left|\psi_{1}^{1}\right|^{2},\left|\psi_{2}^{1}\right|^{2}\right)=\left(\sin ^{2}(\alpha / 2), \cos ^{2}(\alpha / 2)\right)$.

By our choice of the angle $\alpha$ (10), we have $\left(\left|\psi_{1}^{1}\right|^{2},\left|\psi_{2}^{1}\right|^{2}\right)=\left(d_{1}, d_{2}\right)$.

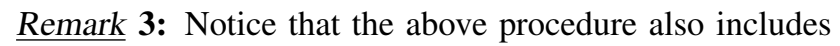
the special cases $\alpha=0, \pi$. If $\alpha=0$ the control goes straight from $(-1,0)$ to $(1,0)$, and then comes back with a semicircle; the wave function at the final time $\left(\tau_{k}^{0} / L\right) T$ is $\psi=(0,1)$ (modulo a phase factor), which means that the state has completely flipped to the eigenstate relative to $\lambda=+1$, in accord with the adiabatic theory. On the contrary, if $\alpha=\pi$ then the path reduces to the segment connecting $(-1,0)$ to the origin followed by the segment that comes back to the initial point. In this case, the final state is $\psi=(1,0)$ (with a phase factor), that is the trajectory comes back to the original state. Notice that in this case any reparametrization $\mu^{\pi}$ leads to the same result.

With our choice of the initial condition the time reparametrization of the control functions involved in the definition of SCBP takes a particularly simple form. However, if the initial condition $\psi^{0}$ is a superposition of states, we can prove that the control functions of the form (8), suitably rescaled in time, still provide an explicit strategy for SCBP. The explicit computations are cumbersome, and we therefore postpone them to a forthcoming paper.

\section{CONCLUSION}

In this paper, we provided a control strategy that permits to steer exactly a two level quantum system presenting a conical singularity to a final state with given probabilities. This strategy can be arbitrarily slowed down, which is important in order to reduce an infinite dimensional quantum system to a two level system as the one considered in this paper, that is then decoupled from the other levels. The tool permitting such a decoupling is the adiabatic theory. The aim of the further research in this direction is to get an approximate controllability result for the general system. 
The main advantages of the proposed strategy are that it does not require the Hamiltonian to depend linearly on the controls, and that it provides an explicit expression of the controls.

\section{REFERENCES}

[1] R. Adami and U. Boscain. Controllability of the Schrödinger equation via intersection of eigenvalues. In Proceedings of the 44th IEEE Conference on Decision and Control, December 12-15, pages 1080$1085,2005$.

[2] F. Albertini and D. D'Alessandro. Notions of controllability for bilinear multilevel quantum systems. IEEE Trans. Automat. Control, 48(8):1399-1403, 2003.

[3] L. Allen and J. H. Eberly. Optical Resonance and Two-Level Atoms. Wiley, New York, 1975.

[4] C. Altafini. Controllability of quantum mechanical systems by root space decomposition of su(n). Journal of Mathematical Physics, 43(5):2051-2062, 2002.

[5] K. Beauchard and J.-M. Coron. Controllability of a quantum particle in a moving potential well. J. Funct. Anal., 232(2):328-389, 2006.

[6] A. Bloch, R. Brockett, and C. Rangan. The controllability of infinite quantum systems and closed subspace criteria. Preprint, 2006.

[7] U. Boscain, T. Chambrion, and G. Charlot. Nonisotropic 3-level quantum systems: Complete solutions for minimum time and minimal energy. Discrete and Continuous Dynamical Systems-B, 5(4):957-990, 2005.

[8] U. Boscain, T. Chambrion, and G. J.-P. On the $\mathrm{k}+\mathrm{p}$ problem for a three-level quantum system: Optimality implies resonance. Journal of Dynamical and Control Systems, 8:547-572, 2002.

[9] U. Boscain, G. Charlot, G. J.-P., S. Guérin, and H.-R. Jauslin. Optimal control in laser-induced population transfer for two- and three-level quantum systems. Journal of Mathematical Physics, 43:2107-2132, 2002.

[10] T. Chambrion, P. Mason, M. Sigalotti, and U. Boscain. Controllability of the discrete-spectrum Schrödinger equation driven by an external field. Annales de l'Institut Henri Poincaré, analyse non linéaire, doi:10.1016/j.anihpc.2008.05.001, 2008.

[11] Y. Colin de Verdière. Construction de laplaciens dont une partie finie (avec multiplicités) du spectre est donnée. In Séminaire sur les équations aux dérivées partielles 1986-1987, pages Exp. No. VII, 6. École Polytech., Palaiseau, 1987.

[12] M. A. Dahleh, A. P. Peirce, and H. Rabitz. Optimal control of quantum-mechanical systems: Existence, numerical approximation, and applications. Phys. Rev. A, 37:4950-4964, 1988.

[13] D. D'Alessandro. Introduction to quantum control and dynamics. Chapman \& Hall/CRC Applied Mathematics and Nonlinear Science Series. Chapman \& Hall/CRC, Boca Raton, FL, 2008.

[14] D. D'Alessandro and M. Dahleh. Optimal control of two-level quantum systems. IEEE Transactions on Automatic Control, 46(6):866876, 2001.

[15] U. Gaubatz, P. Rudecki, M. Becker, S. Schiemann, M. Kulz, and K. Bergmann. Population switching between vibrational levels in molecular beams. Chem. Phys. Lett., 149:463, 1988.

[16] S. Guérin and H. R. Jauslin. Control of quantum dynamics by laser pulses: Adiabatic floquet theory. Advances in Chemical Physics, 125, 2003.

[17] P. Hübler, J. Bargon, and S. J. Glaser. Nuclear magnetic resonance quantum computing exploiting the pure spin state of para hydrogen. J. Chem. Phys., 113(6):2056-2059, 2000.

[18] N. Khaneja, R. Brockett, and S. J. Glaser. Time optimal control in spin systems. Phys. Rev. A, 63, 2001.

[19] M. Mirrahimi. Lyapunov control of a quantum particle in a decaying potential. Ann. Inst. H. Poincaré Anal. Non Linéaire, 26(5):1743-1765, 2009.

[20] V. Nersesyan. Growth of Sobolev norms and controllability of the Schrödinger equation. Comm. Math. Phys., 290(1):371-387, 2009.

[21] M. A. Nielsen and I. L. Chuang. Quantum computation and quantum information. Cambridge University Press, Cambridge, 2000.

[22] V. Ramakrishna, H. Rabitz, M. Salapaka, M. Dahleh, and A. Peirce. Controllability of molecular systems. Phys. Rev. A, 62:960-966, 1995.
[23] M. Shapiro and P. Brumer. Principles of the Quantum Control of Molecular Processes. Principles of the Quantum Control of Molecular Processes, pp. 250. Wiley-VCH, Feb. 2003.

[24] S. Teufel. Adiabatic perturbation theory in quantum dynamics, volume 1821 of Lecture Notes in Mathematics. Springer-Verlag, Berlin, 2003.

[25] L. P. Yatsenko, S. Guérin, and H. R. Jauslin. Topology of adiabatic passage. Phys. Rev. A, 65(043407):7 pages, 2002. 